\title{
Silicon Drift Detectors: Limitations for Throughput and Resolution
}

\author{
Jens Rafaelsen ${ }^{1}$
}

1. EDAX Inc, A Division of Ametek, Materials Analysis Division, Mahwah, NJ

Silicon Drift Detector (SDD) based Energy Dispersive X-ray Spectroscopy (EDS) systems have undergone significant improvements since their inception [1] further improving the dramatic speed, resolution, and low energy sensitivity gains they offer over the older $\mathrm{Si}(\mathrm{Li})$ systems. To identify the limitations and bottlenecks of a given system, the three primary components of the system should be considered: The Si chip, the preamplifier, and the pulse processor.

As shown in Figure 1, the voltage output of the preamplifier will have discrete steps corresponding to individual charge clouds generated by X-rays hitting the detector with each individual step exhibiting a module characteristic charge collection time. The charge collection time is the first limitation in a system as the rise time gives the upper limit for the number of X-rays that can be analyzed per second, i.e. limiting the detection speed.

The preamplifier signal feeds into the DPP which essentially digitizes the signal and extracts the X-ray energy. This is typically done by applying various filters and generating a triangular shaped pulse as illustrated in Figure 2 (left). The total pulse time (rise time + gap time + fall time) is often referred to as the shaping time and the shortest shaping time will again impose a limitation on how many X-rays that can be analyzed per second. The resolution of a given EDS detector is defined as the Full Width Half Maximum (FWHM) of the Mn Ka peak which is affected by the Fano broadening and the electronic noise in the system which can generally be considered as being proportional to the leakage current, $1 / \mathrm{f}$ noise and thermal noise:

Noise $\propto I_{l e a k}+C^{2}+k T \frac{C^{2}}{\tau}$,

where $C$ is the capacitance, $k$ is the Boltzmann constant, $T$ is the temperature, and $\tau$ is the shaping time. As can be seen from the equation, lowering the capacitance will significantly reduce the noise in the system and consequently improve the resolution and low energy performance while going to shorter shaping times increases the noise and leads to broader peaks. Figure 2 (right) illustrates the difference between two different detector systems where one utilizes a CUBE [2] preamplifier exhibiting lower capacitance resulting in a significant reduction in FWHM, especially at the fast shaping times.

The ultimate throughput/speed of a given system is determined by a combination of the fastest shaping time available in the DPP and the charge collection time. If the shaping time is faster than the charge collection time, part of the charge will not be assigned correctly in the DPP resulting in low energy tailing. This is an artifact that can sometimes be seen on large area detectors where the charge collection time depends on how far from the anode the charge cloud was created. Figure 3 illustrates this by comparing Mn spectra using different size limiting apertures on a slow charge collection time module. The long shaping time allows for full charge collection regardless of exposed area, but at the fast shaping time tailing is seen to increase with area exposure. 


\section{References:}

[1] L. Strüder et al, Mikrochimica Acta, Supplement 15 (1998), p. 11.

[2] L. B. C. Fiorini et al, IEEE Nuclear Science Symposium Conference Record (2011) p. 1972.
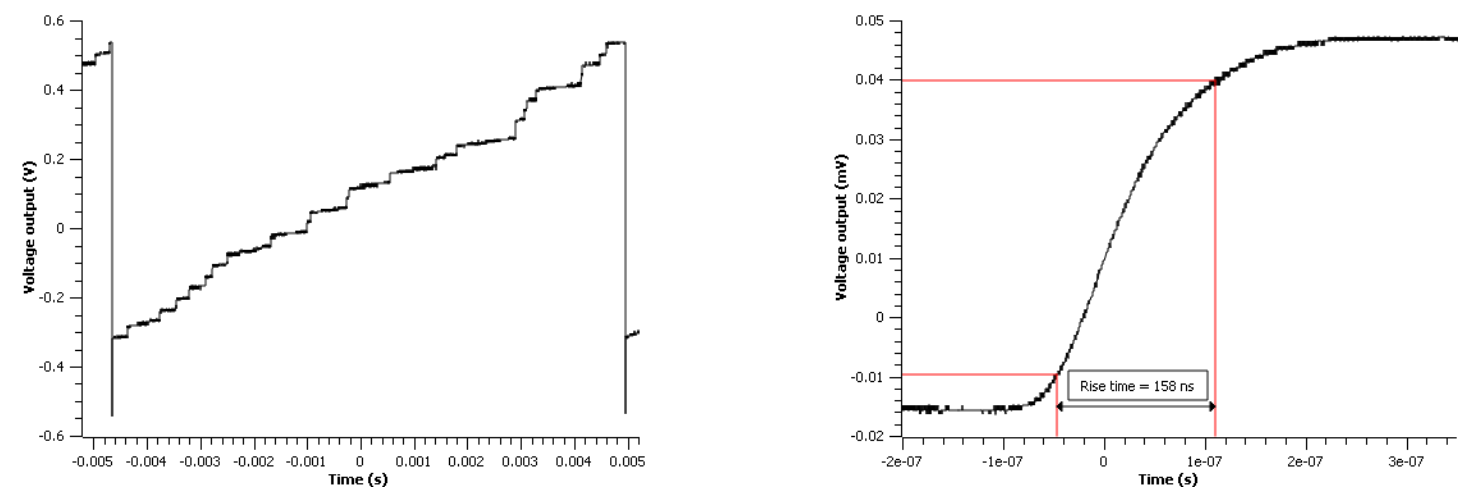

Figure 1. (Left) Voltage output from the preamplifier showing steps corresponding to detected X-rays and reset pulses. (Right) single voltage step showing the detector rise time using the 10\%-90\% criteria.
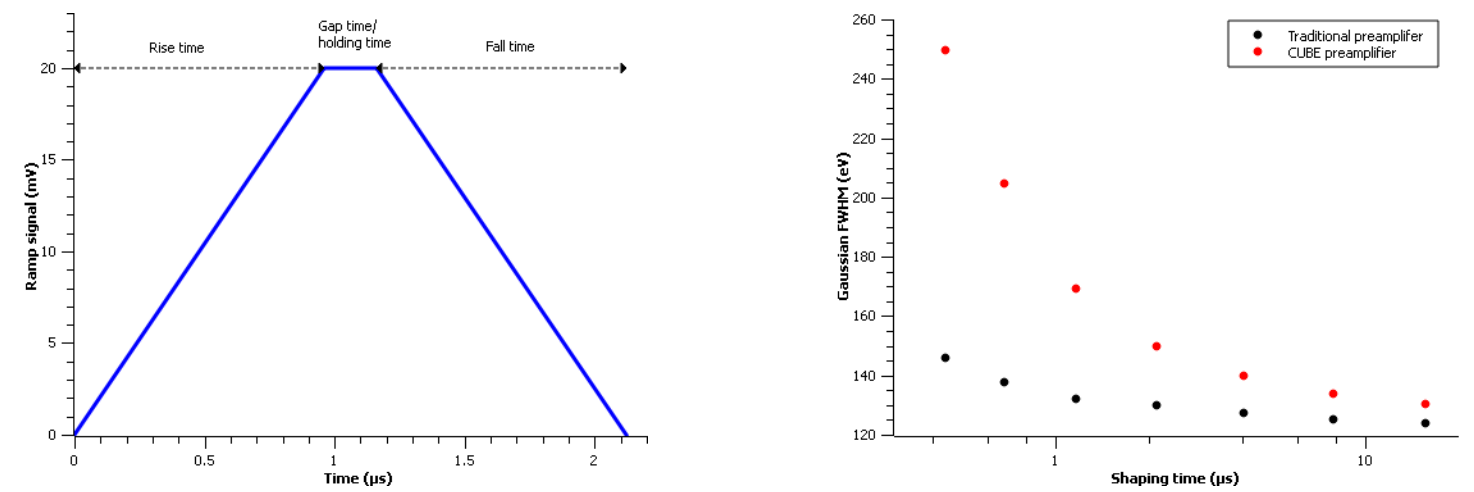

Figure 2. (Left) Illustration of pulse processor ramp signal and timings. (Right) Mn Ka FWHM as a function of shaping time for two different generation detectors with comparable active area.
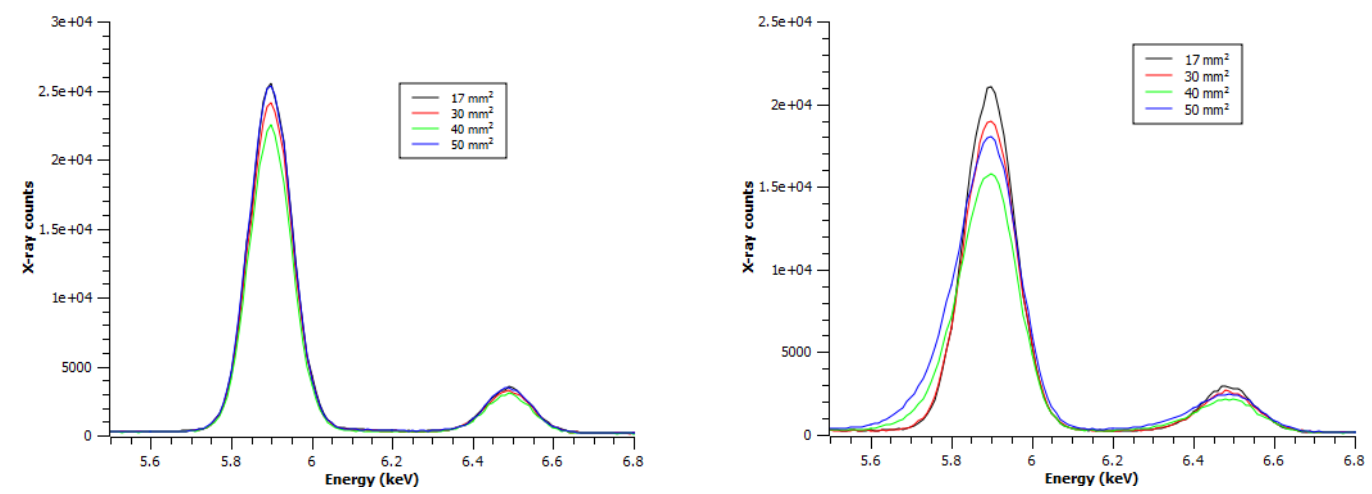

Figure 3. Non-normalized Mn spectra with varying aperture sizes for the $15.56 \square \mathrm{s}$ (Left) and $0.44 \mathrm{~ns}$ shaping time (Right). 